[CONTRIBUTIONS FROM THE SHEFFIFID LABORATORY OF YALE UNIVERSITY.]

\title{
HYDANTOINS: THE PREPARATION OF HYDANTOIN FROM HIPPURIC ACID.
}

[TWENTY-FIFTH PAPER.]

By TRBAT B. JOHNSON AND ROBHRT BENGIS.

Received July 24, 1913.

The new method of synthesizing hydantoin III, which we describe in this note, is recommended when glycocoll and potassium cyanate are not available, and it is desired to obtain a small amount of this compound quickly and without much labor. The method involves only two operations and the only reagents necessary are hippuric acid I, ammonium thiocyanate and chloroacetic acid.

Hippuric acid I is first converted into 2-thio-3-benzoylhydantoin II according to the method of Johnson and Nicolet. ${ }^{1}$ The yield of this compound is always about $85-90 \%$ of the theoretical, if the operation is properly conducted. Furthermore, the hydantoin can be purified easily. The next operation involves a desulfurization and hydrolysis of this thiohydantoin II. This can easily be accomplished by digestion of the hydantoin with an aqueous solution of chloroacetic acid. Benzoic acid and hydantoin III are formed smoothly, and the yield of the latter compound is generally about $70 \%$ of the theoretical. The two phases of the transformation may be represented as follows:

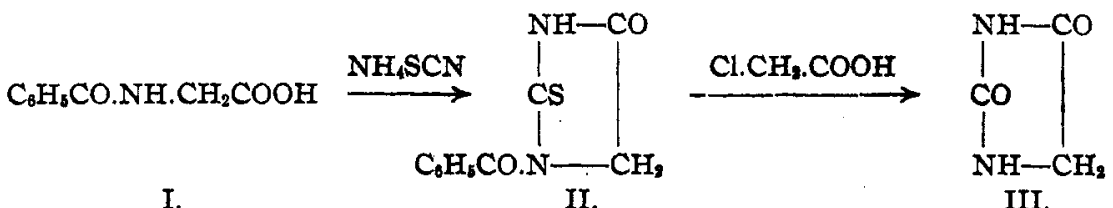

We have applied the method successfully several times, and the transformations are apparently applicable for all $\alpha$-acyl amino acids.

Experimental Part.

2-Thio-3-benzoylhydantoin,

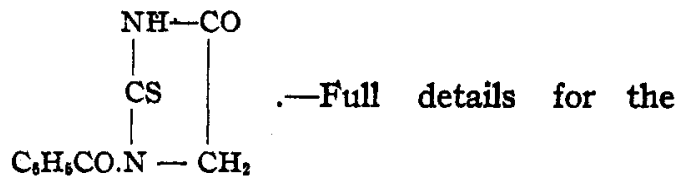

preparation of this compound are given in previous papers from this laboratory. ${ }^{2}$

Conversion of the Acylthiohydantoin into Hydantoin.-Ten grams of the thiohydantoin are suspended in $25-40 \mathrm{cc}$. of water, in which are dissolved 5 grams of chloroacetic acid. The flask is then connected with a return condenser and the mixture digested on a sand bath until the thiohydantoin

${ }^{1}$ This Journal, 33, 1973; Am. Chem. J., 49, 202.

2 Johnson and Nicolet, Loc. cit. 
completely dissolves. This is generally accomplished in $1.5^{-2}$ hours. After the hydantoin is decomposed the solution is then concentrated to a volume of about $20-25 \mathrm{cc}$. and cooled, when the benzoic acid will separate. Practically $70 \%$ of the benzoic acid will deposit here and can be separated by filtration. After removal of the benzoic acid the filtrate is then concentrated to a thick syrup, by evaporation at $100^{\circ}$, and finally diluted with absolute alcohol. Pure hydantoin will separate immediately in a colorless, granular condition and melting, without further purification, at $217^{\circ}$. This is then separated by filtration and the filtrate combined with its volume of alcohol and the evaporation repeated. A syrup is generally obtained which can then be triturated with alcohol, when more hydantoin will be obtained. From ten grams of the thiohydantoin 3.0 grams of pure hydantoin can easily be obtained. This corresponds to a yield of $70 \%$ of the theoretical.

NEW IAVEN, CONA.

[CONTRIBUtIONS FROM THE SHEFFIELD LABORATORY OF YALE UNIVERSITY.]

\section{HYDANTOINS: SYNTHESES OF 3-METHOXY - 4-HYDROXY - PHENYLALANINE AND 3,4-DIMETHOXYPHENYLALANINE.}

[TWENTY-SIXTH PAPER.]

BY TREAT B. JohNSON AND ROBERT BENGIS.

Received July $24,1913$.

The recent and important contributions, by Pictet and his co-workers, to our knowledge of the papaverine and berberine groups of the opium alkaloids have aroused an interest in hydroxy derivatives of the two aromatic $\alpha$-amino acids, namely, phenylalanine and tyrosine. The naturally occurring bases of these two groups are derivatives of isoquinoline, and the results obtained by Pictet show that there is a genetic relationship between these alkaloids and aromatic $\alpha$-amino acids. In other words, the evidence is strong, from a chemical standpoint, that the precursors of these alkaloids are amino acids, which result from the decomposition of plant proteins.

Isoquinoline compounds can easily be obtained from the aromatic $\alpha$-amino acids or their bacterial decomposition products, namely, the corresponding $\beta$-amines. Phenylalanine (I) and tyrosine, for example, interact smoothly with methylal and formaldehyde, under proper conditions, with formation of isoquinoline carboxylic acids (II), and Pictet and Spengler ${ }^{1}$ express the assumption, in their paper describing these changes, that the isoquinoline alkaloids may be produced in plants by similar condensations. It is of special interest to note here that Beattie ${ }^{2}$ actually found $\mathrm{I}$-oxyisoquinoline-3-carboxylic acid (III) in the form of

${ }^{1}$ Bir., 44, 2030. See also Wellisch, Biochem. $Z, 49,173$ (1913).

${ }^{2}$ Am. Chem. I., 40,415, 